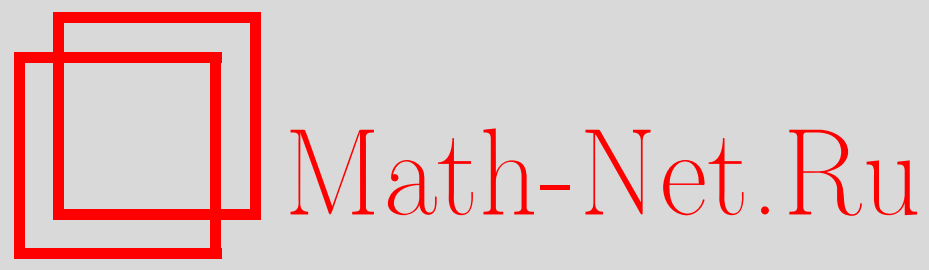

Р. С. Юлмухаметов, О целых функциях с заданным асимптотическим поведением, Функи. анализ и его прил., 1998, том 32, выпуск 3, 50-61

DOI: https://doi.org/10.4213/faa422

Использование Общероссийского математического портала MathNet.Ru подразумевает, что вы прочитали и согласны с пользовательским соглашением

http://www . mathnet.ru/rus/agreement

Параметры загрузки:

IP : 34.229 .45 .116

26 апреля 2023 г., 18:33:05

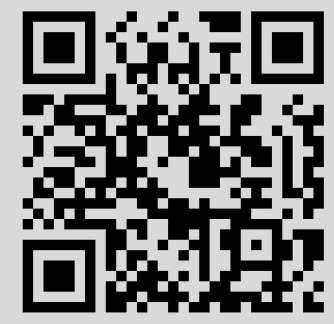


Функииональный анализ и его приложения

1998, т. 32, вып. 3, с. 50-61

УДК 517.574

\section{О целых функциях с заданным асимптотическим поведением}

(c) 1998. Р. С. Юлмухаметов

В данной работе рассматривается вопрос о существовании целых функций с наперед заданным поведением в бесконечности. Этот вопрос являлся объектом исследований многих авторов. В работах А. Пфлюгера и Б. Я. Левина были построены целые функции одной переменной, имеющие заданную индикатрису при заданном уточненном порядке. Для функций многих переменных аналогичные факты доказаны Мартино [10] и Кисельманом [9]. В 1969 г. В. С. Азарин [1] получил общий результат: для любой субгармонической функции $u$ конечного порядка $\rho$ существует целая функция $f$, удовлетворяющая соотношению

$$
|u(z)-\ln | f(z)||=o\left(|z|^{\rho}\right), \quad|z| \rightarrow \infty .
$$

В дальнейшем в работах Ю. И. Мельника [7], М. Л. Содина, Ю. И. Любарского [8] и автора результат Азарина развивался в направлении уточнения асимптотики. В [2] показано, что целая функция $f$ может быть построена так, чтобы в правой части формулы (*) можно было поставить $O(\ln |z|)$. Аналог теоремы Азарина в случае многих переменных доказан Сигурдсоном [3] и, независимо, автором [4]. Результат Сигурдсона с помощью теоремы Азарина (см. [5, теорема 4.2.2]) можно сформулировать так: для любой плюрисубгармонической функции $u$ конечного порядка $\rho$ существует целая функция $f$, для которой выполняется $(*)$. В [4] показано, что при тех же условиях в $(*)$ можно добиться точности $O\left(|z|^{4 \rho / 5}\right)$.

Соотношения вида $(*)$ выполняются лишь вне некоторых исключительных множеств, покрывающих особенности функций $u$ и $\ln |f|$. Краткое обсуждение вопроса об оценке размеров этих исключительных множеств мы проведем в конце статьи.

Результаты о существовании целых функций с заданной асимптотикой находят применения в теории рядов экспонент, уравнениях свертки и др.

В данной работе предлагается принципиально новый метод построения целых функций с заданным поведением в бесконечности, и этот метод применяется для построения целой функции $F(\lambda)$, удовлетворяющей соотношениям

$$
c \rho(\lambda)^{s} e^{u(\lambda)} \leqslant|F(\lambda)| \leqslant C(1+|\lambda|)^{k} e^{u(\lambda)},
$$

где $u$ - заданная выпуклая функция в $\mathbb{C}^{p}$ с некоторыми дополнительными свойствами и $\rho(\lambda)$ - расстояние от точки $\lambda$ до множества нулей функции $F$. 
Этот результат по точности совпадает с наиболее точными результатами для функций одной переменной (см. [2]).

Предварительно докажем две вспомогательные леммы.

Пусть $v(t) \in C^{2}\left(\mathbb{C}^{p}\right)$ - строго выпуклая функция и

$$
K(\lambda, z)=\int e^{\langle\lambda, t\rangle+\langle\bar{z}, \bar{t}\rangle-2 v(t)} d t, \quad \lambda, z \in \mathbb{C}^{p}
$$

где

$$
\langle\lambda, t\rangle=\sum_{k=1}^{n} \lambda_{k} t_{k}
$$

ЛЕмма 1. Предположим, ито для любого $m \in \mathbb{N}$ выполнено условие

$$
e^{m|t|-2 v(t)}\left(\left|\frac{\partial v(t)}{\partial t}\right|+1\right) \rightarrow 0 \quad n p u|t| \rightarrow \infty
$$

где $\partial v(t) / \partial t=\left(\partial v(t) / \partial t_{1}, \ldots, \partial v(t) / \partial t_{p}\right)$. Тог $\partial a$

$$
|K(\lambda, z)|^{2} \leqslant \frac{1}{|\lambda-z|^{4}} \int e^{2 \operatorname{Re}\langle\lambda, t\rangle-2 v(t)} \Delta v(t) d t \int e^{2 \operatorname{Re}\langle z, t\rangle-2 v(t)} \Delta v(t) d t
$$

где $\Delta v(t)$ - оператор Лапласа от функиии $v$.

ДокАЗАТЕльство. Из представления $\left(\lambda_{j} \neq z_{j}\right)$

$$
K(\lambda, z)=\int e^{2 \operatorname{Re}\langle\lambda, t\rangle-2 v(t)} \frac{\partial}{\partial \bar{t}_{j}} \frac{e^{\langle\bar{z}-\bar{\lambda}, \bar{t}\rangle}}{\bar{z}_{j}-\bar{\lambda}_{j}} d t
$$

интегрированием по частям с учетом условия (1) получим

$$
\left(\bar{z}_{j}-\bar{\lambda}_{j}\right) K(\lambda, z)=-\int e^{\langle\lambda, t\rangle+\langle\bar{z}, \bar{t}\rangle-2 v(t)} \frac{\partial}{\partial \bar{t}_{j}}(2 \operatorname{Re}\langle\lambda, t\rangle-2 v(t)) d t
$$

Аналогичным образом получаем

$$
\begin{aligned}
\left|\bar{z}_{j}-\bar{t}_{j}\right|^{2} K(\lambda, z)=2 & \int e^{\langle\lambda, t\rangle+\langle\bar{z}, \bar{t}\rangle-2 v(t)} \frac{\partial^{2} v(t)}{\partial t_{j} \partial \bar{t}_{j}} d t \\
& -\int e^{\langle\lambda, t\rangle+\langle\bar{z}, \bar{t}\rangle-2 v(t)}\left(z_{j}-2 \frac{\partial v(t)}{\partial t_{j}}\right)\left(\bar{\lambda}_{j}-2 \frac{\partial v(t)}{\partial \bar{t}_{j}}\right) d t
\end{aligned}
$$

Для сокращения записи введем обозначение

$$
E(\zeta, t, v)=e^{2 \operatorname{Re}\langle\zeta, t\rangle-2 v(t)}
$$


Воспользуемся далее неравенством Коши-Буняковского:

$$
\begin{aligned}
& \left|z_{j}-\lambda_{j}\right|^{4}|K(\lambda, z)|^{2} \leqslant 8 \int E(\lambda, t, v) \frac{\partial^{2} v(t)}{\partial t_{j} \partial \bar{t}_{j}} d t \cdot \int E(z, t, v) \frac{\partial^{2} v(t)}{\partial t_{j} \partial \bar{t}_{j}} d t \\
& +2 \int E(\lambda, t, v)\left|\lambda_{j}-2 \frac{\partial v(t)}{\partial t_{j}}\right|^{2} d t \cdot \int E(z, t, v)\left|z_{j}-2 \frac{\partial v(t)}{\partial t_{j}}\right|^{2} d t .
\end{aligned}
$$

Поскольку

$$
\int E(\lambda, t, v)\left|\lambda_{j}-2 \frac{\partial v(t)}{\partial t_{j}}\right|^{2} d t=\int\left(\bar{\lambda}_{j}-2 \frac{\partial v(t)}{\partial \bar{t}_{j}}\right) \frac{\partial}{\partial t_{j}} E(\lambda, t, v) d t,
$$

то, интегрируя по частям, с учетом условия (1) имеем

$$
\int E(\lambda, t, v)\left|\lambda_{j}-\frac{\partial v(t)}{\partial t_{j}}\right|^{2} d t=2 \int E(\lambda, t, v) \frac{\partial^{2} v(t)}{\partial t_{j} \partial \bar{t}_{j}} d t
$$

Подставляя это равенство в (2), получим

$$
\left|z_{j}-\lambda_{j}\right|^{2}|K(\lambda, z)| \leqslant 4\left(\int E(\lambda, t, v) \frac{\partial^{2} v}{\partial t_{j} \partial \bar{t}_{j}} d t\right)^{1 / 2}\left(\int E(z, t, v) \frac{\partial^{2} v}{\partial t_{j} \partial \bar{t}_{j}} d t\right)^{1 / 2} .
$$

Суммируя полученные неравенства по всем $j=1, \ldots, p$ и применив к правой части неравенство Коши-Буняковского, получим утверждение леммы 1.

Коротко напомним некоторые факты из теории выпуклых функций.

Пусть $u \in C^{2}\left(\mathbb{R}^{n}\right)$ - строго выпуклая функция. Введем обозначения

$$
\begin{gathered}
u^{\prime}(x)=\left(\frac{\partial u(x)}{\partial x_{1}}, \ldots, \frac{\partial u(x)}{\partial x_{n}}\right): \mathbb{R}^{n} \rightarrow \mathbb{R}^{n}, \\
U^{\prime \prime}(x)=\left(\frac{\partial^{2} u(x)}{\partial x_{k} \partial x_{m}}\right), \quad v(y)=\sup _{x}((x, y)-u(x)) .
\end{gathered}
$$

Из строгой выпуклости функции $u$ следует положительность $\operatorname{det} U^{\prime \prime}(x)$, и по теореме об обратной функции отображение $u^{\prime}$ является локальным диффеоморфизмом. Если $x=x(y)$ - точка достижения супремума в определении функции $v$, то $u^{\prime}(x(y)) \equiv y$ и, таким образом, $x(y) \in C^{1}$. Дифференцируя тождество $v(y) \equiv(y, x(y))-u(x(y))$, получаем $x(y) \equiv v^{\prime}(y)$, т.е. $v^{\prime}$ - отображение, обратное к $u^{\prime}$, и $v \in C^{2}$. Матрица

$$
V^{\prime \prime}(y)=\left(\frac{\partial^{2} v(y)}{\partial y_{k} \partial y_{m}}\right)
$$

обратна к матрице $U^{\prime \prime}(x(y))$; значит, если $\lambda_{1}, \ldots, \lambda_{n}$ - собственные числа матрицы $U^{\prime \prime}(x)$, то $\lambda_{1}^{-1}, \ldots, \lambda_{n}^{-1}$ - собственные числа матрицы $V^{\prime \prime}(y(x))$. Предположим, что для некоторого $\alpha>1$ имеет место соотношение

$$
0<C_{1}(1+|x|)^{\alpha-2}|a|^{2} \leqslant\left(U^{\prime \prime}(x) a, a\right) \leqslant C_{2}(1+|x|)^{\alpha-2}|a|^{2}, \quad x, a \in \mathbb{R}^{n} .
$$


Выражение $f(x) \asymp g(x)$ в дальнейшем будет означать, что

$$
0<C_{1} \leqslant \frac{f(x)}{g(x)} \leqslant C_{2}, \quad|x| \rightarrow \infty .
$$

Наше условие, принимающее вид

$$
\frac{\left(U^{\prime \prime}(x) a, a\right)}{|a|^{2}} \asymp(1+|x|)^{\alpha-2}
$$

для функции $v$ означает

$$
\frac{\left(V^{\prime \prime}(y) a, a\right)}{|a|^{2}} \asymp(1+|x(y)|)^{2-\alpha} .
$$

Рассматривая сужение функции $u$ на лучи, исходящие из начала координат, получим

$$
u(x) \asymp(1+|x|)^{\alpha}, \quad\left|u^{\prime}(x)\right| \asymp(1+|x|)^{\alpha-1}, \quad\left|v^{\prime}(y)\right| \asymp(1+|y|)^{\beta-1},
$$

где число $\beta$ определяется из условия $1 / \alpha+1 / \beta=1$. Таким образом,

$$
\frac{\left(V^{\prime \prime}(y) a, a\right)}{|a|^{2}} \asymp(1+|y|)^{\beta-2}, \quad \Delta v(y) \asymp(1+|y|)^{\beta-2} .
$$

Лемма 2. Пусть $u(z) \in C^{2}\left(\mathbb{C}^{p}\right)$ - строго вылуклая функиия, удовлетворяюшая соотношению

$$
\frac{\left(U^{\prime \prime}(z) a, a\right)}{|a|^{2}} \asymp(1+|z|)^{\alpha-2}, \quad a \in \mathbb{R}^{2 p}, z \in \mathbb{C}^{p},|z| \rightarrow \infty,
$$

әде $\alpha>1$ - некоторое число. Положим

$$
v(t)=\sup (\operatorname{Re}\langle z, \bar{t}\rangle-u(\bar{z})), \quad K(\lambda, z)=\int e^{\langle\lambda, t\rangle+\langle\bar{z}, \bar{t}\rangle-2 v(t)} d t, \quad \lambda, z \in \mathbb{C}^{p}
$$

тогда функиия $v$ удовлетворяет условиям леммы 1 и выполняется соотношение

$$
\frac{|K(\lambda, z)|^{2}}{K(\lambda, \lambda) K(z, z)} \leqslant \frac{C(1+|\lambda|)^{2-\alpha}(1+|z|)^{2-\alpha}}{|\lambda-z|^{4}}, \quad \lambda, z \in \mathbb{C}^{p}
$$

ДокАЗАтЕльство. Функция $v$ удовлетворяет оценке (1) в силу (3). Для доказательства неравенства (5) достаточно получить оценку

$$
\int e^{2 \operatorname{Re}\langle\lambda, t\rangle-2 v(t)} \Delta v(t) d t \leqslant C_{1}(1+|\lambda|)^{2-\alpha} K(\lambda, \lambda) .
$$


Зафиксируем точку $\lambda \in \mathbb{C}^{p}$. Пусть $q>0$. Заметим, что

$$
u(z)=\sup _{t}(\operatorname{Re}\langle z, t\rangle-v(t))
$$

и положим

$$
U(\lambda, q)=\left\{t \in \mathbb{C}^{p}: 2 v(t)+2 u(\lambda)-2 \operatorname{Re}\langle\lambda, t\rangle<q\right\},
$$

а через $\alpha_{\lambda}(q)$ обозначим $2 p$-мерный объем множества $U(\lambda, q)$. Как известно [6], $\left(\alpha_{\lambda}(q)\right)^{1 / 2 p}$ - возрастающая вогнутая функция. Поэтому $\alpha_{\lambda}(q) \leqslant$ $\alpha_{\lambda}(1) q^{2 p}$, когда $q>1$. Интегрированием по частям получим

$$
\begin{aligned}
\int E(\lambda, t, v) e^{-2 u(\lambda)} d t & =\int_{0}^{\infty} e^{-q} d \alpha_{\lambda}(q)=\int_{0}^{\infty} \alpha_{\lambda}(q) e^{-q} d q \\
& \leqslant \int_{1}^{\infty} \alpha_{\lambda}(1) e^{-q} q^{2 p} d q+\int_{0}^{1} \alpha_{\lambda}(1) e^{-q} d q \\
& \leqslant \alpha_{\lambda}(1) \int_{0}^{\infty} e^{-q}(1+q)^{2 p} d q \leqslant \alpha_{\lambda}(1)(2 p) ! e .
\end{aligned}
$$

С другой стороны,

$$
\int E(\lambda, t, v) e^{-2 u(\lambda)} d t \geqslant \int_{U(\lambda, 1)} E(\lambda, t, v) e^{-2 u(\lambda)} d t \geqslant e^{-1} \alpha_{\lambda}(1) .
$$

Таким образом, для любой выпуклой функции имеем оценку

$$
e(2 p) ! \alpha_{\lambda}(1) \geqslant \int e^{2 \operatorname{Re}\langle\lambda, t\rangle-2 v(t)-2 u(\lambda)} d t \geqslant e^{-1} \alpha_{\lambda}(1)
$$

Из (4) следует, что при достаточно большом $M$ функция

$$
\tilde{v}(t)=v(t)-\frac{\beta-2}{2} \ln \left(|t|^{2}+M\right)
$$

выпукла в $\mathbb{R}^{2 p}$ и также удовлетворяет соотношениям из (4). Положим $\tilde{u}(z)=$ $\sup (\operatorname{Re}\langle z, t\rangle-\tilde{v}(t))$. В силу (4) имеем

$$
\begin{aligned}
\int e^{2 \operatorname{Re}\langle\lambda, t\rangle-2 v(t)} \Delta v(t) d t & \leqslant C_{1} \int(|t|+M)^{\beta-2} e^{2 \operatorname{Re}\langle\lambda, t\rangle-2 v(t)} d t \\
& \leqslant C_{1} \int e^{2 \operatorname{Re}\langle\lambda, t\rangle-2 \tilde{v}(t)} d t .
\end{aligned}
$$

Отсюда и из соотношения (6), примененного к паре $\tilde{u}$ и $\tilde{v}$, вытекает неравенство

$$
\int e^{2 \operatorname{Re}\langle\lambda, t\rangle-2 v(t)} \Delta v(t) d t \leqslant C_{2} e^{\tilde{u}(\lambda)} \widetilde{\alpha}_{\lambda}(1)
$$


где $\widetilde{\alpha}_{\lambda}(1)$ есть $2 p$-мерный объем множества

$$
\widetilde{V}(\lambda, 1)=\{t: 2 \tilde{v}(t)+2 \tilde{u}(\lambda)-2 \operatorname{Re}\langle\lambda, t\rangle<1\} .
$$

Поэтому

$$
\int e^{2 \operatorname{Re}\langle\lambda, t\rangle-2 v(t)} \Delta v(t) d t \leqslant C_{2} e \int_{\widetilde{V}(\lambda, 1)} e^{2 \operatorname{Re}\langle\lambda, t\rangle-2 \tilde{v}(t)} d t
$$

Пусть $t=\tilde{t}(\lambda)$ - точка, для которой $\tilde{u}(\lambda)=\operatorname{Re}\langle\lambda, t\rangle-\tilde{v}(t)$. Как уже отмечалось, при этом $\lambda=2 \partial \tilde{v}(\tilde{t}(\lambda)) / \partial t$. Следовательно,

$$
\tilde{V}(\lambda, 1)=\left\{t: \tilde{v}(t)-\tilde{v}(\tilde{t}(\lambda))-2 \operatorname{Re}\left\langle\frac{\partial \tilde{v}(\tilde{t}(\lambda))}{\partial t}, t-\tilde{t}(\lambda)\right\rangle<\frac{1}{2}\right\} .
$$

По формуле Тейлора

$$
\tilde{v}(t)-\tilde{v}(\tilde{t}(\lambda))-2 \operatorname{Re}\left\langle\frac{\partial \tilde{v}(\tilde{t}(\lambda))}{\partial t}, t-\tilde{t}(\lambda)\right\rangle=\frac{1}{2}\left(\widetilde{V}^{\prime \prime}(\tau)(t-\tilde{t}(\lambda)), t-\tilde{t}(\lambda)\right)
$$

здесь $\tau$ - точка между $t$ и $\tilde{t}(\lambda)$, а $t-\tilde{t}(\lambda)$ рассматривается как элемент из $\mathbb{R}^{2 p}$. По выбору числа $M$ для функции $\tilde{v}$ выполняются соотношения (4). В силу этого для точек $t$, лежащих на сфере $|t-\tilde{t}(\lambda)|=x(1+|\tilde{t}(\lambda)|)^{1-\beta / 2}$, справедлива оценка

$$
\left(\widetilde{V}^{\prime \prime}(\tau)(t-\tilde{t}(\lambda)), t-\tilde{t}(\lambda)\right) \asymp x^{2}(1+|\tau|)^{\beta-2}(1+|\tilde{t}(\lambda)|)^{2-\beta} .
$$

Заметим, что $|\tau| /|\tilde{t}(\lambda)| \rightarrow 1$ при фиксированном $x$. Это значит, что существуют числа $0<x_{1}<x_{2}<\infty$, такие, что выпуклое множество $\widetilde{V}(\lambda, 1)$ удовлетворяет включениям

$$
B\left(\tilde{t}(\lambda), x_{1}(1+|\tilde{t}(\lambda)|)^{1-\beta / 2}\right) \subset \tilde{V}(\lambda, 1) \subset B\left(\tilde{t}(\lambda), x_{2}(1+|\tilde{t}(\lambda)|)^{1-\beta / 2}\right) .
$$

Отсюда и из (7) вытекает оценка

$$
\begin{aligned}
\int E(\lambda, t, v) \Delta v(t) d t & \leqslant C \int_{B\left(\tilde{t}(\lambda), x_{2}(1+|\tilde{t}(\lambda)|)^{1-\beta / 2}\right)} E(\lambda, t, \tilde{v}) d t \\
& \leqslant C(1+|\tilde{t}(\lambda)|)^{1-\beta / 2} \int E(\lambda, t, v) d t \\
& =C(1+|\tilde{t}(\lambda)|)^{1-\beta / 2} K(\lambda, \lambda) .
\end{aligned}
$$

Так как $\tilde{v}^{\prime}(\tilde{t}(\lambda))=\lambda$, то из оценки $(3)$, примененной к функции $\tilde{v}$, имеем

$$
|\tilde{t}(\lambda)|^{\beta-1} \leqslant C|\lambda|
$$


Следовательно,

$$
\int E(\lambda, t, v) \Delta v(t) d t \leqslant C(|\lambda|+1)^{(\beta-2) /(\beta-1)} K(\lambda, \lambda)=C(|\lambda|+1)^{2-\alpha} K(\lambda, \lambda) .
$$

Лемма 2 доказана.

Теорема 1. Пусть $u \in C^{2}\left(\mathbb{C}^{p}\right)-$ строго выпуклал функиия, удовлетворяющал условию леммь 2. Тогда существует челая функиия $F(\lambda)$, которал для некоторых постоянных $A_{1}, A_{2}, s$ удовлетворяет соотношению

$$
A_{1}\left(\rho_{0}(\lambda)\right)^{s} e^{u(\lambda)} \leqslant|F(\lambda)| \leqslant A_{2}(1+|\lambda|)^{(\alpha-2) p^{2}} e^{u(\lambda)}, \quad \lambda \in \mathbb{C}^{p},
$$

¿де

$$
\rho_{0}(\lambda)=\min \left\{\frac{1}{1+|\lambda|}, \inf _{z: F(z)=0}|\lambda-z|\right\} .
$$

ДокаЗАТЕльство. Возьмем в пространстве $\mathbb{C}^{p}$ систему точек $z_{k}, k=$ $1,2, \ldots$, удовлетворяющую следующим двум условиям:

(C1) шары $B\left(z_{k}, d\left(1+\left|z_{k}\right|\right)^{1-\alpha / 2}\right)$ попарно не пересекаются;

(C2) шары $B\left(z_{k}, 4 d\left(1+\left|z_{k}\right|\right)^{1-\alpha / 2}\right)$ покрывают все пространство.

Число $d$ будет выбрано ниже. Через $v$ обозначим функцию, сопряженную по Юнгу с функцией $u(\bar{\lambda}) /(2 p)$, и определим функцию $K(\lambda, z)$, как в лемме 2 . Положим

$$
F(\lambda)=\sum_{k=1}^{\infty} \frac{K\left(\lambda, z_{k}\right)^{2 p}}{K\left(z_{k}, z_{k}\right)^{p}}
$$

Зафиксируем точку $\lambda \in \mathbb{C}^{p}$ и через $I(\lambda)$ обозначим множество индексов $k$, для которых

$$
\left|\lambda-z_{k}\right| \geqslant 2 d\left(1+\left|z_{k}\right|\right)^{1-\alpha / 2} .
$$

Так как функция $|\lambda-z|^{-4 p}$ субгармонична по $z$ в $\mathbb{C}^{p} \backslash\{\lambda\}$, то по утверждению леммы 2 для $k \in I(\lambda)$ выполняется неравенство

$$
\frac{\left|K\left(\lambda, z_{k}\right)\right|^{2 p}}{K\left(z_{k}, z_{k}\right)^{p} K(\lambda, \lambda)^{p}} \leqslant \frac{C^{p}(1+|\lambda|)^{p(2-\alpha)}}{w_{p} d^{2 p}} \int_{B\left(z_{k}, d\left(1+\left|z_{k}\right|\right)^{1-\alpha / 2}\right)} \frac{d w(z)}{|\lambda-z|^{4 p}},
$$

где $w_{p}$ - объем единичного шара в $\mathbb{C}^{p}$. Суммируя эти неравенства, получим, что

$$
\sum_{I(\lambda)} \frac{\left|K\left(\lambda, z_{k}\right)\right|^{2 p}}{K\left(z_{k}, z_{k}\right)^{p} K(\lambda, \lambda)^{p}} \leqslant \frac{C^{p}(1+|\lambda|)^{p(2-\alpha)}}{w_{p} d^{2 p}} \int_{|\lambda-z| \geqslant d q(1+|\lambda|)^{1-\alpha / 2}} \frac{d w(z)}{|\lambda-z|^{4 p}}
$$

где $q$ - достаточно маленькое число, зависящее только от $d$ и $\alpha>1$. Отсюда вытекает оценка

$$
\sum_{k \in I(\lambda)} \frac{\left|K\left(\lambda, z_{k}\right)\right|^{2 p}}{K\left(z_{k}, z_{k}\right)^{p}} \leqslant \frac{C^{p}}{2 p d^{4 p} q^{2 p}} K(\lambda, \lambda)^{p}
$$


В силу условия (C1) количество индексов $k$, не лежащих в $I(\lambda)$, конечно, причем ограничено числом $N=N(\alpha, p)$, не зависящим от $\lambda$. Поскольку

$$
|K(\lambda, z)|^{2} \leqslant K(\lambda, \lambda) K(z, z)
$$

TO

$$
\sum_{k \notin I(\lambda)} \frac{\left|K\left(\lambda, z_{k}\right)\right|^{2 p}}{K\left(z_{k}, z_{k}\right)^{p}} \leqslant N(\alpha, p) K(\lambda, \lambda)^{p} .
$$

Таким образом, получаем верхнюю оценку

$$
|F(\lambda)| \leqslant C K(\lambda, \lambda)^{p}, \quad \lambda \in \mathbb{C}^{p} .
$$

Так же, как и неравенство (9), для любого $m \in \mathbb{N}$ может быть получена оценка

$$
\sum_{k \neq m} \frac{\left|K\left(z_{m}, z_{k}\right)\right|^{2 p}}{K\left(z_{k}, z_{k}\right)^{p}} \leqslant \frac{C^{p}}{2 p d^{4 p}} K\left(z_{m}, z_{m}\right)^{p},
$$

где постоянная $C$ не зависит от $m$. Выберем число $d$ так, чтобы $C^{p} /\left(2 p d^{4 p}\right)=$ $1 / 2$. Тогда

$$
\left|F\left(z_{m}\right)\right| \geqslant K\left(z_{m}, z_{m}\right)^{p}-\sum_{k \neq m} \frac{\left|K\left(z_{m}, z_{k}\right)\right|^{2 p}}{K\left(z_{k}, z_{k}\right)^{p}} \geqslant \frac{1}{2} K\left(z_{m}, z_{m}\right)^{p} .
$$

Из соотношения (6) следует, что

$$
K(\lambda, \lambda) \asymp e^{u(\lambda) / p} \alpha_{\lambda}(1),
$$

где $\alpha_{\lambda}(1)$ есть $2 p$-мерный объем множества

$$
V(\lambda, 1)=\left\{t: 2 v(t)+p^{-1} u(\lambda)-2 \operatorname{Re}\langle\lambda, t\rangle<1\right\} .
$$

Из неравенства (8) видно, что

$$
\alpha_{\lambda}(1) \asymp|t(\lambda)|^{(2-\beta) p} \asymp\left|u^{\prime}(\lambda)\right|^{(2-\beta) p} \asymp|\lambda|^{(\alpha-2) p} .
$$

Таким образом, оценки (10) и (11) означают выполнение условий

$$
\begin{array}{ll}
|F(\lambda)| \leqslant C(1+|\lambda|)^{(\alpha-2) p^{2}} e^{u(\lambda)}, & \lambda \in \mathbb{C}^{p}, \\
|F(\lambda)| \geqslant c(1+|\lambda|)^{(\alpha-2) p^{2}} e^{u(\lambda)}, & \lambda=z_{m}, m \in \mathbb{N} .
\end{array}
$$

ЗАмЕчАНИЕ. По ходу доказательства мы воспользовались тем, что для некоторого $q=q(\alpha, d)$ имеет место включение

$$
\bigcup_{k \in I(\lambda)} B\left(z_{k}, d\left(1+\left|z_{k}\right|\right)^{1-\alpha / 2}\right) \subset \mathbb{C}^{n} \backslash B\left(\lambda, d q(1+|\lambda|)^{1-\alpha / 2}\right) .
$$


Чтобы убедиться в этом, возьмем точку $w$ из объединения шаров в левой части. Это значит, что для некоторого $j$ имеют место соотношения

$$
\left|\lambda-z_{j}\right| \geqslant 2 d\left(1+\left|z_{j}\right|\right)^{1-\alpha / 2}, \quad\left|w-z_{j}\right| \leqslant d\left(1+\left|z_{j}\right|\right)^{1-\alpha / 2} .
$$

1) Пусть $\alpha \leqslant 2$; тогда, поскольку

$$
|w-\lambda| \geqslant\left|z_{j}-\lambda\right|-\left|z_{j}-w\right| \geqslant d\left(1+\left|z_{j}\right|\right)^{1-\alpha / 2},
$$

то для $z_{j},\left|z_{j}\right| \geqslant|\lambda| / 2$,

$$
|w-\lambda| \geqslant d q_{1}(1+|\lambda|)^{1-\alpha / 2},
$$

где

$$
q_{1}=\inf \left\{\left(\frac{1+\left|z_{j}\right|}{1+|\lambda|}\right)^{1-\alpha / 2}, j \in I(\lambda),\left|z_{j}\right| \geqslant \frac{|\lambda|}{2}\right\}>0 .
$$

Для $\left|z_{j}\right| \leqslant|\lambda| / 2$ и для рассматриваемого $w$ имеем

$$
|w| \leqslant\left|z_{j}\right|+d\left(1+\left|z_{j}\right|\right)^{1-\alpha / 2} \leqslant \frac{|\lambda|}{2}+d\left(1+\frac{|\lambda|}{2}\right)^{1-\alpha / 2} .
$$

Поэтому

$$
|w-\lambda| \geqslant|\lambda|-|w| \geqslant|\lambda| / 2-d(1+|\lambda|)^{1-\alpha / 2} .
$$

Так как $1-\alpha / 2<1$, то отсюда видно, что

$$
|w-\lambda| \geqslant d(1+|\lambda|)^{1-\alpha / 2}, \quad|\lambda| \geqslant R=R(\alpha, d) .
$$

Наконец, для $\lambda,|\lambda| \leqslant R$, из $(* *)$ получим

$$
\frac{|w-\lambda|}{d(1+|\lambda|)^{1-\alpha / 2}} \geqslant \frac{1}{(1+R)^{1-\alpha / 2}}=q_{2}
$$

Остается положить $q=\min \left(q_{1}, q_{2}, 1\right)$.

2) Пусть $\alpha>2$. Воспользуемся $(* *)$ и для $z_{j},\left|z_{j}\right| \leqslant 2(|\lambda|+d)$, получим

$$
|w-\lambda| \geqslant d q_{1}(1+|\lambda|)^{1-\alpha / 2}
$$

где

$$
q_{1}=\inf \left\{\left(\frac{1+\left|z_{j}\right|}{1+|\lambda|}\right)^{1-\alpha / 2}, j \in I(\lambda),\left|z_{j}\right| \leqslant 2(|\lambda|+d)\right\}>0 .
$$

Для $\left|z_{j}\right|>2(|\lambda|+d)$ и для рассматриваемого $w$ имеем

$$
|w| \geqslant\left|z_{j}\right|-d\left(1+\left|z_{j}\right|\right)^{1-\alpha / 2} \geqslant\left|z_{j}\right|-d \geqslant 2|\lambda|+d .
$$


Поэтому

$$
|w-\lambda| \geqslant|w|-|\lambda| \geqslant|\lambda|+d \geqslant d \geqslant d(1+|\lambda|)^{1-\alpha / 2} .
$$

Положим $q=\min \left(q_{1}, 1\right)$.

ЛЕмма 3. Пусть функция $f$ голоморфна в круге $|\zeta|<4$ и

$$
\ln |f(\zeta)| \leqslant M, \quad|\zeta|<4, \quad \ln |f(0)| \geqslant m .
$$

Тогда существуют постолннье $C=C(M, m)$ u $s=s(M, m)$, такие, ито выполняется соотношение

$$
|f(\zeta)| \geqslant C \rho(\zeta)^{s}
$$

где $\rho(\zeta)$ - расстояние от точки $\zeta$ до множества нулей функции $f$.

Доказательство этой леммы, основанное на лемме Гельмгольца, проводится стандартными рассуждениями.

По условию теоремы для любых $z_{k}$ и $z \in B\left(z_{k}, 16 d\left(1+\left|z_{k}\right|\right)^{1-\alpha / 2}\right)$ имеем оценку

$$
\left|u(z)-u\left(z_{k}\right)-2 \operatorname{Re}\left\langle\partial u\left(z_{k}\right) / \partial z, z-z_{k}\right\rangle\right|=\frac{1}{2}\left(U^{\prime \prime}\left(z^{\prime}\right)\left(z-z_{k}\right), z-z_{k}\right) \leqslant \text { const }
$$

где const не зависит от индекса $k$. Рассмотрим функции

$$
\widetilde{F}_{k}(\lambda)=F(\lambda)\left(1+\left|z_{k}\right|\right)^{(2-\alpha) p^{2}} \exp \left(-u\left(z_{k}\right)-2 \operatorname{Re}\left\langle\partial u\left(z_{k}\right) / \partial z, z-z_{k}\right\rangle\right) .
$$

Из соотношения (12) следует, что функции

$$
f_{k}(\zeta)=\widetilde{F}_{k}\left(z_{k}+2 \zeta \omega d\left(1+\left|z_{k}\right|\right)^{1-\alpha / 2}\right), \quad|\zeta| \leqslant 4,
$$

где $\omega \in \mathbb{C}^{p},|\omega|=1$, удовлетворяют условиям леммы 3 , причем постоянные $M, m$ можно выбрать не зависящими от индекса $k$. Поэтому имеют место оценки

$$
\left|\widetilde{F}_{k}(\lambda)\right| \geqslant C \rho(\lambda)^{s}, \quad \lambda \in B\left(z_{k}, 4 d\left(1+\left|z_{k}\right|\right)^{1-\alpha / 2}\right) .
$$

Отсюда и из условия (С2) следуют требуемые нижние оценки.

Теорема 1 доказана.

В заключение приведем результат теоремы 1 в привычном виде, а именно как асимптотическое соотношение типа $(*)$, и получим некоторые оценки возникающих исключительных множеств.

Лемма 4. Функиия $F(\lambda)$ в теореме 1 удовлетворяет соотношению

$$
|u(\lambda)-\ln | F(\lambda)||=O(\ln |\lambda|), \quad|\lambda| \rightarrow \infty, \lambda \notin E .
$$

При этом исключительное множество $Е$ может быть покрыто иарами $B\left(\lambda_{k}, r_{k}\right)$ таким образом, что

$$
\sum_{k} r_{k}^{2 p-1}<\infty
$$


ДокАЗАТЕЛЬСтво. Как следует из (3), функция $u(\lambda)$ имеет порядок роста $\alpha$, т.е. $u(\lambda) \leqslant$ const $(|\lambda|+1)^{\alpha}$. Следовательно, для $\ln |F(\lambda)|$ имеем такую же верхнюю оценку:

$$
\ln |F(\lambda)| \leqslant \operatorname{const}(|\lambda|+1)^{\alpha} .
$$

Пусть $\mu(t)$ есть $(2 p-1)$-мерный объем дивизора функции $F$ в шаре $B(0, t)$. Из верхней оценки функции $F$ получим (см. [11])

$$
\mu(t) \leqslant \operatorname{const}(|\lambda|+1)^{2 p+\alpha-1} .
$$

Положим

$$
S_{n}=\left\{z: F(z)=0,2^{n}<|z| \leqslant 2^{n+1}\right\} .
$$

Возьмем некоторое $\gamma<0$. По лемме Хеймана о покрытиях [12] можно выбрать точки $z_{1}, \ldots, z_{m}$ из множества $S_{n}$ так, чтобы шары

$$
B\left(z_{k}, 2^{n \gamma}\right), \quad k=1, \ldots, m
$$

покрывали множество $S_{n}$ и каждая точка пространства попадала не более чем в $\kappa_{p}$ шаров, где $\kappa_{p}$ зависит лишь от размерности $p$. Положим

$$
E_{n}=\bigcup_{k=1}^{m} B\left(z_{k}, 2^{n \gamma}\right),
$$

и пусть $\left|E_{n}\right|$ обозначает объем множества $E_{n}$. Очевидно, что

$$
2^{2 p n \gamma} m \leqslant \text { const } \kappa_{p}\left|E_{n}\right| \leqslant \text { const } 2^{2 n \gamma}\left|S_{n}\right|,
$$

где $\left|S_{n}\right|$ есть $(2 p-2)$-мерный объем множества $S_{n}$. Отсюда, учитывая оценку (14), получим

$$
\left(2^{n \gamma}\right)^{2 p-1} m \leqslant \text { const } 2^{n \gamma} 2^{n(2 p+\alpha-1)} .
$$

Далее положим $\gamma=-(2 p+\alpha)$ :

$$
\left(2^{n \gamma}\right)^{2 p-1} m \leqslant \text { const } 2^{-n},
$$

причем const не зависит от $n$. Если теперь мы рассмотрим множества

$$
E_{n}^{\prime}=\bigcup_{k=1}^{m} B\left(z_{k}, 2^{n \gamma+1}\right),
$$

где точки $z_{1}, \ldots, z_{m}$ для каждого $n$ выбраны так, как изложено выше, то множество

$$
E=\bigcup_{n=0}^{\infty} E_{n}^{\prime}
$$


в силу (15) удовлетворяет условиям леммы 4. Ясно также, что вне множества $E$ функция $F$ удовлетворяет соотношению (13).

\section{ЛИТЕРАТУРА}

1. Азарин B. C. О лучах вполне регулярного роста целой функции. Матем. сб., 79, No. 4, 463-476 (1969).

2. Юлмухаметов Р. С. Аппроксимация субгармонических функций. Anal. Math., 11, 257-282 (1985).

3. Sigurdsson $R$. Growth properties of analytic and plurisubharmonic functions of finite order. Lund University, 1984.

4. Юлмухаметов $P$. C. Целые функции многих переменных с заданным поведением в бесконечности. Изв. РАН, сер. матем., 60, № 4, 205-224 (1996).

5. Азарин B. C. Об асимптотическом поведении субгармонических функций конечного порядка. Матем. сб., 108 (150), № 3, 147-167 (1979).

6. Погорелов A. В. Внешняя геометрия выпуклых поверхностей. Наука, М., 1969.

7. Мельник Ю. И. О представлении функций рядами Дирихле в замкнутом круге. Матем. сб., 97, № 4, 493-502 (1975).

8. Любарский Ю. И., Содин М. Л. Аналог функции типа синуса для выпуклых областей. ФТИНТ АН УССР, Харьков, 17, 42-52 (1986).

9. Kiselman $C$. O. On entire funtions of exponential type and indicators of analytic functionals. Acta Math., 117, 1-35 (1967).

10. Martineau $A$. Indicatrices de croissance des fonctions entieres de $N$-variables. Invent. Math., 2, № 1, 81-86 (1966).

11. Ронкин Л. И. Введение в теорию целых функций многих переменных. Наука, M., 1971.

12. Ландкоф Н. С. Основы современной теории потенциала. Наука, М., 1966.

Башкирский государственный университет,

Поступило в редакцию математический факультет

27 марта 1996 г.

email: yavdat@bgua.bashkiria.su 